\title{
Contribuições ao conhecimento dos gêneros Elanela e Nocheta (Heteroptera, Pentatomini) ${ }^{1}$
}

\author{
Jocélia Grazia $^{2}$
}

\begin{abstract}
The male of Elanela hevera Rolston et alii, 1980 and the female of Nocheta adda Rolston et alii, 1980 are described for the first time. A complete study of external genitalia for both sexes of $\mathbf{E}$. hevera and $\mathbf{N}$. adda is also presented.
\end{abstract}

\section{INTRODUÇÃO}

Os gêneros Elanela e Nocheta foram descritos por Rolston, McDonald e Thomas Jr., 1980 e incluídos na seção três de Pentatomini caracterizada pela presença de um tubérculo mediano no 30 . esternito abdominal aposto a uma reentrânsia na margem posterior do metasterno.

Ambos são monotípicos: Elanela hevera Rolston et alii, 1980 é baseada numa única fêmea coletada em Iquitos, Peru; Nocheta adda Rolston et alii, 1980 é baseada num único macho procedente da amazônia brasileira.

A disponibilidade de exemplares de ambos os sexos de $E$. hevera e $N$. $a d d a$ e, considerando que os machos da primeira espécie e as fêmeas da segunda eram até então desconhecidos, levou-nos a apresentar um estudo detalhado da genitália destas espécies.

Todos os exemplares examinados de $N$. adda e parte dos exemplares de $E$. hevera foram obtidos no Museu de Leiden (Holanda) através do Dr. P. H. van Doesburg a quem agradecemos; os demais exemplares de $E$. hevera procedem do Museu Goeldi (Belém) e da coleção da CEPLAC (Belém), estes últimos coletados pelo Dr. J. A. Garcia em cacaueiro, com "choque de B.H.C. $12 \%$ " nas plantações de Bragantina e Guajarina, Estado do Pará, os quais foram recebidos para identificação.

As medidas estão expressas em milímetros e a metodologia adotada segue GRAZIA (1983).

1 Trabalho apresentado no XV Congresso Brasileiro de Zoologia, Curitiba-PR, 31/01 a 05/ $02 / 88$.

2 Departamento de Zoologia, Universidade Federal do Rio Grande do Sul, Av. Paulo Gama s/no 90049 Porto Alegre-RS, Brasil. Bolsista do CNPq. 
Elanela hevera Rolston, McDonald e Thomas Jr. 1980:125-126

Descrição geral de acordo com ROLSTON et al. (1980). Os machos, ao contrário das fêmeas que têm o abdome imaculado, apresentam uma mancha ferrugínea oblonga, desde o 4 o até o 70 urosternito, ao longo da linha mediana longitudinal. Segundo artículo antenal com uma linha ferrugínea láteroventral nítida; nas fêmeas examinadas esta linha é descontínua, às vezes inaparente; quanto ao tamanho, ora o $2 \%$ artículo é maior que o 39 , ora o 39 predomina sobre o 2 ?, ou ainda, ambos são iguais, sendo o 5 \% sempre maior que o 4 ?.

Macho - Medidas: comprimento da cabeça $1,26(1,12-1,36)$; largura da cabeça 2,26 (2,2 - 2,32); distância interocular $1,18(1,12-1,2)$; distância interocelar $0,5(0,48-0,56)$; comprimento diante dos olhos $0,5(0,48-0,56)$. Comprimento dos artículos antenais I 0,36 , II 1,04 $(0,92$ - 1,16), III $1,06(0,96$ $1,16)$, IV $1,82(1,72-1,96)$, V $(1,84-2,08)$. Comprimento do pronoto 1,78 $(1,64-1,88)$; largura do pronoto $4,97(4,8-5,2)$; comprimento do escutelo 3,31 $(3,16$ - 3,44$)$; largura do escutelo $3,21(3-3,32)$; comprimento total $5,98(5,89$. $6,16)$.

Genitália - Pigóforo globóide achatando-se dorsoventralmente em direção posterior. Parede dorsal do pigóforo profundamente escavada de cada lado do bordo dorsal (BD) (Fig. 1). Margem ven tral (MV) do pigóforo com escavação semilunar no terço apical, essa área recoberta por longos pêlos (Fig. 2). Proctiger (X segmento) bastante desenvolvido, ultrapassando os ângulos póstero-laterais do pigóforo, com a margem posterior recortada em "V" profundo (Figs. 1, 2). Parâmeros (Par) espatulares, estreitados na base e ampliando-se em direção apical (Fig. 3). Phallus: Processus capitati (pc) bastante desenvolvidos em relação as placas basais do aparelho articular (Figs. 4,5). Conjuntiva ausente; vésica (V) globóide, ductus seminis distalis (Dsd) muito curto, gonopóro secundário (GS) abrindo-se dorsalmente. (Figs. 5, 6). Phallotheca $(\mathrm{Ph})$ piriforme medindo o dobro do comprimento do aparelho articular, ao longo da linha mediana longitudinal, com 1 par de processos basais ( $\operatorname{PrPh} 1)$. Abertura da phallotheca pósterodorsal, estreita (Fig. 4).

Fêmea - Genitália: Margem posterior do esternito VII uniformemente côncava sobre os laterotergitos (La8) e gonocoxitos 8 (Gc8). Laterotergitos 8 (La8) com espiráculos. Gonocoxitos 8 (Gc8) subquadrangulares, margem posterior truncada, bordos suturais justapostos. Comprimento do $\mathrm{Gc} 8$ ao longo dos bordos suturais com quase o dobro do comprimento dos laterotergitos 9 (La9), estes últimos sublanceolados, muito pouco ultrapassando a banda que une ventralmente os laterotergitos 8. Margem posterior dos gonocoxitos 9 (pseudoesternito - Gc9) nitidamente escavada em pequeno "U" aberto (Fig. 7). Vias genitais ectodérmicas: ductus receptaculi (DR), na região posterior a área vesicular, muito longo, com quase duas vezes o comprimento do ductus na região anterior a área vesicular. Área vesicular ampla, bastante desenvolvida com mais de quatro vezes o comprimento dos laterotergitos 9 . Gonapófises 9 (G9) com cerca de duas vezes o comprimento dos gonocoxitos 9; chitinelipsen ausentes. Capsula seminalis (CS) esférica com um longo processo digitiforme que ultrapassa a crista anular anterior (Caa) (Fig. 8). 


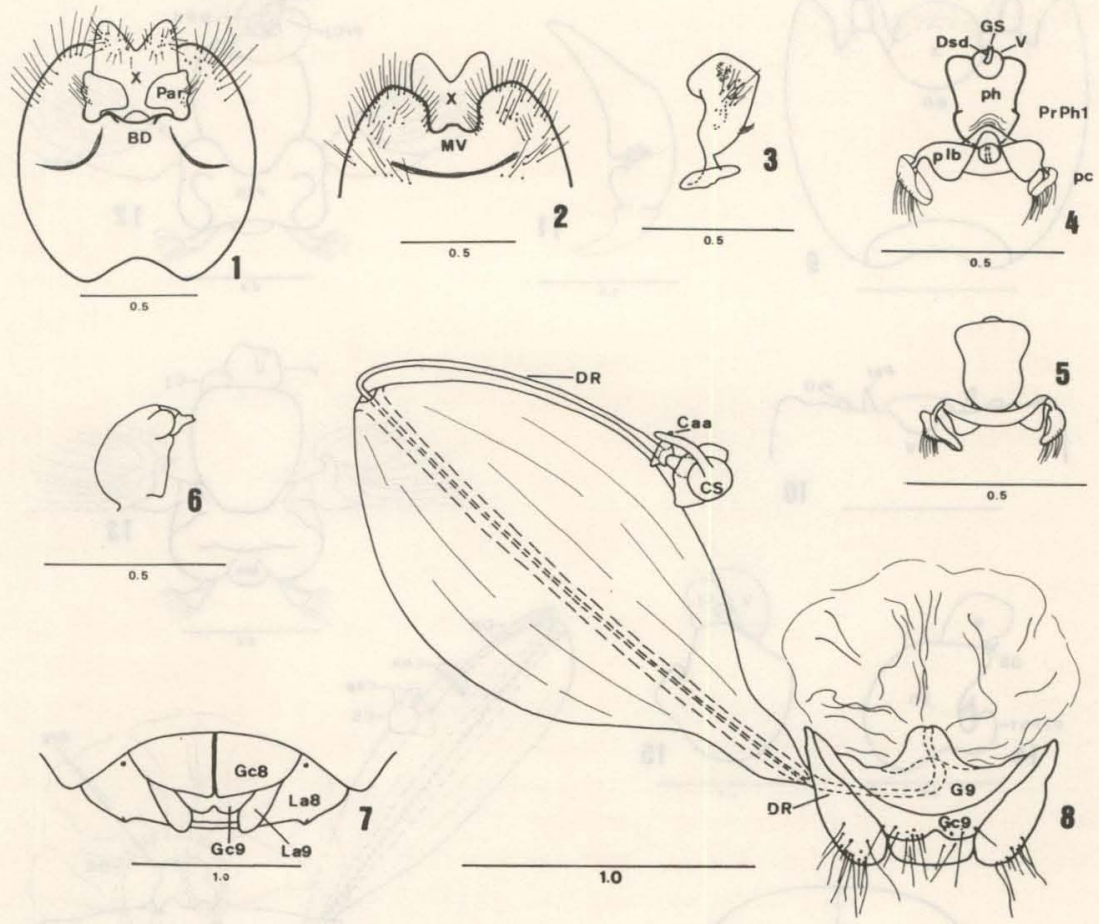

FIGS. 1 -8 -Elanela hevera Rolston et alii, 1980. 1, Pigóforo, vista dorsal; 2, Margem ventral do pigóforo; 3, Parâmero esquerdo, vista látero-dorsal; 4, Phallus, dorsal; 5, Phallus, ventral; 6, Phallus, lateral; 7, Placas genitais da fêmea, vista ventral; 8, Laterotergitos, gonocoxitos e gonapófises do 9 ? segmento e receptaculum seminis. $(\mathrm{BD}=$ bordo dorsal, $\mathrm{DR}=$ ductus receptaculi, $\mathrm{Dsd}=$ ductus seminis distalis, $\mathrm{Caa}=$ crista anular anterior, $\mathrm{CS}=$ capsula seminalis, $\mathrm{Gc} 8=$ gonocoxitos $8, \mathrm{Gc} 9=$ gonocoxitos $9, \mathrm{G} 8=$ gonapófises $8, \mathrm{G} 9=$ gonapófises $9, \mathrm{GS}=$ gonóporo secundário, $\mathrm{La} 8=$ laterotergitos 8 , La9 = laterotergitos $9, \mathrm{MV}=$ margem ventral, $\mathrm{Par}=$ parâmeros, $\mathrm{pc}=$ processus capita$\mathrm{ti}, \mathrm{ph}=$ phallotheca, $\mathrm{plb}=$ placas basais, $\mathrm{PrPh} 1=$ processus phallothecae $1, \mathrm{~V}=$ vésica, $\mathrm{X}=10$. segmento). Escalas em milímetros.

Material examinado - SURINAME: 2 machos e 1 fêmea - Paramaribo, 23/VI/1975, P.H.v.Doesburg, Museu de Leiden; 1 fêmea - Paramaribo, 14/VII/ 1975, P.H.v Doesburg, Museu de Leiden. BRASIL: Amapá: 1 fêmea - Porto Platon, 21/VII/1961, J. \& B. Bechyné, Museu Goeldi; 1 fêmea - Serra do Navio, 18/VII/1961, J. \& B. Bechyné, Museu Goeldi. Pará: 1 macho - km 90 Rod. Transamazônica, 15/III/1980, A.C. Mendes, Coleção CEPLAC; 1 fêmea - km 97 Rod. Transamazônica, 28/I/1980, A.C. Mendes, Coleção CEPLAC; 1 macho - 
Revta bras. Zool.
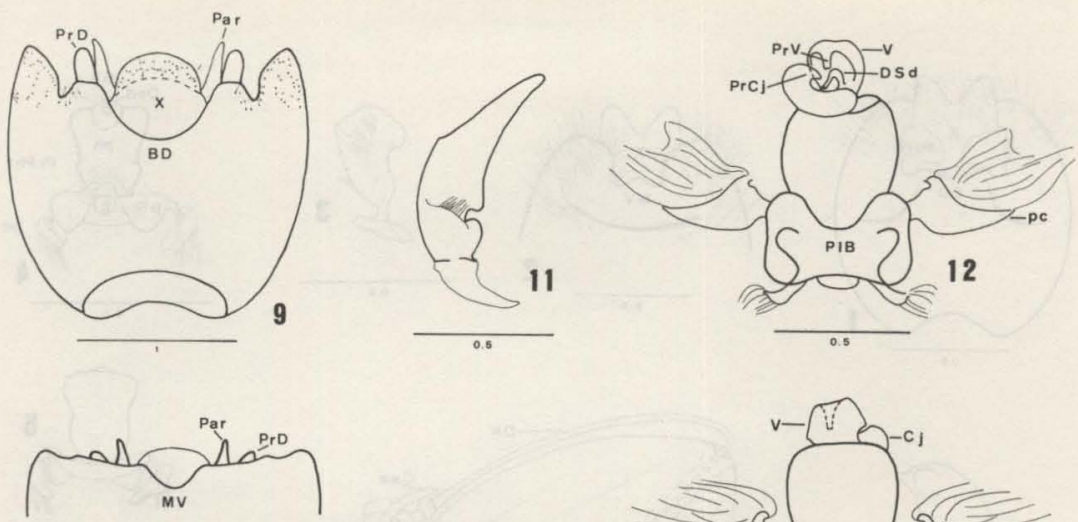

10
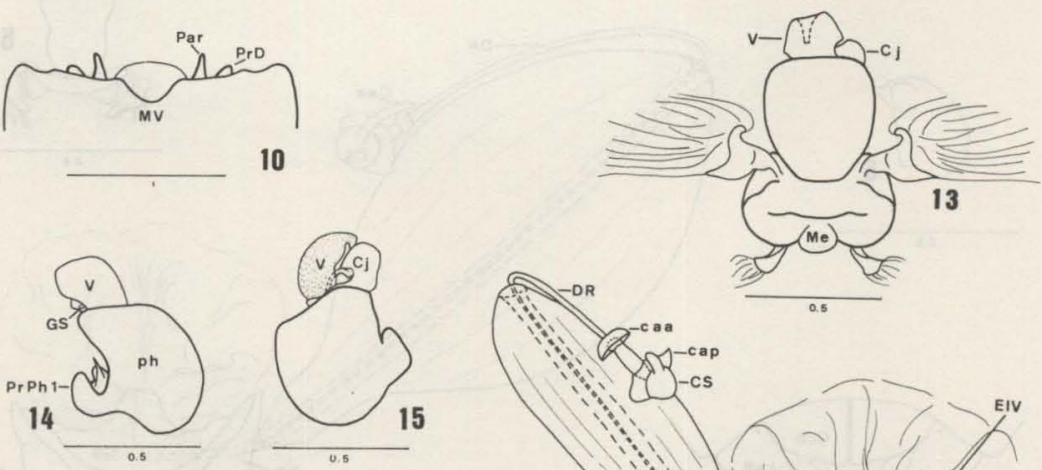

15
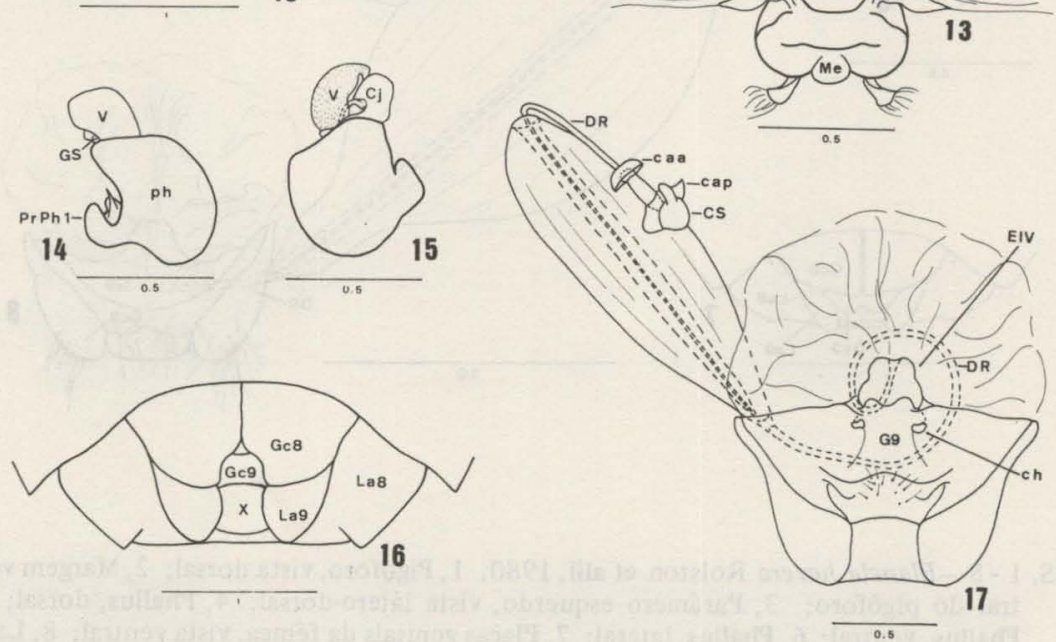

FIGS. 9 - 17 - Nocheta adda Rolston et alii, 1980. 9, Pigóforo, vista dorsal; 10, margem ventral do pigóforo; 11, parâmero esquerdo, vista lateral ex terna; 12, Phallus dorsal; 13, Phallus ventral; 14, Phallus, vista lateral esquerda; 15, Phallus, vista lateral direita; 16, Placas genitais da fêmea; 17, Laterotergitos, gonocoxitos e gonapófises do 9.o segmento e receptaculum seminis. $(\mathrm{BD}=$ bordo dorsal, $\mathrm{DR}=$ ductus receptaculi, DSd = ductus seminis distalis, $\mathrm{Caa}=$ crista anular anterior, $\mathrm{Cap}=$ crista anular posterior, $\mathrm{ch}=$ chitinelipsen, $\mathrm{cj}=$ conjuntiva, $\mathrm{CS}=$ capsula seminalis, $\mathrm{EIV}=$ espessamento da íntima vaginal, $\mathrm{Gc} 8=$ gonocoxitos $8, \mathrm{Gc} 9=$ gonocoxitos $9, \mathrm{G} 9=$ gonapófises 9 , $\mathrm{GS}=$ gonóporo secundário, $\mathrm{La} 8=$ laterotergitos $8, \mathrm{La} 9=$ laterotergitos $9, \mathrm{Me}=$ membranblase, $\mathrm{MV}=$ margem ventral, $\mathrm{Par}=$ parâmeros, $\mathrm{pc}=$ processus capitati, $\mathrm{ph}=\mathrm{phal}$ loteca, $\mathrm{P} 1 \mathrm{~B}=$ placas basais, $\operatorname{PrD}=$ processos do diafragma, $\operatorname{PrC}=$ processo da conjuntiva, $\operatorname{PrPh} 1$ = processus phallothecae $1, \mathrm{~V}=$ vésica, $\mathrm{X}=10$ ? segmento). Escalas em milímetros. 
Altamira, Rod. Transamazônica km 90, 10/III/1979, A.C. Mendes, Coleção CEPLAC; 1 macho e 2 fêmeas - em cacaueiro, 1977/78, J.A. Garcia, Coleção CEPLAC; Amazonas: 1 macho - Itacoatiara, 15/VI/1952, Orlando M. Rego, Museu Nacional; 1 fêmea - Tapurucuara, Rio Negro, 9/II/1963, J. \& B. Bechyné, Museu Goeldi.

\section{Nocheta adda Rolston, McDonald \& Thomas Jr, 1980}

Nocheta adda Rolston, McDonald e Thomas Jr. 1980:129-130

Macho - Genitália: Pigóforo globóide; bordo dorsal (BD) moderadamente côncavo sobre o proctiger (X) e sinuado de cada lado, em pequeno "V" aberto (Fig. 9). Margem ventral (MV) menos recortada que o bordo dorsal, também côncava em "U" aberto, no meio (Fig. 10). Parede do diafragma, na taça genital, com 1 par de processos digitiformes (PrD) bem desenvolvidos, justapostos aos parâmeros (Par), porém menores, em comprimento, que estes (Figs. 9, 10). Parâmeros (Par) aciculares na metade posterior, metade basal com uma reentrância no lado ventral (Fig. 11). Placas basais (P1B) do aparelho articular com cerca da metade do comprimento do phallus ao longo da linha mediana longitudinal: processus capitati (pc) muito amplos, quase tão longos quanto o phallus (Figs. $12,13)$. Phallotheca ( $\mathrm{ph})$, com abertura posterior, com 1 par de processos basais, no lado dorsal, bem desenvolvidos processus phallothecae 1 ( $\mathrm{PrPh} 1)$, entre eles uma projeção acicular. Tanto os processos quanto a projeção acicular são mais facilmente observados em vista lateral. (Fig. 14).

$\mathrm{O}$ conjunto de vésica $(\mathrm{V})$ e conjuntiva $(\mathrm{Cj})$ está disposto assimetricamente em relação ao plano longitudinal da phallotheca (Figs. 12 a 15). Conjuntiva (Cj) com um processo de formato acicular voltado para o lado direito, ápice dirigido para dentro quase encostando no ductus seminis distalis (DsD) (Fig. 12, 15).

Vésica (V) em forma de capacete, situada mais para o lado esquerdo do phallus, junto ao bordo posterior interno um processo digitiforme truncado no ápice (PrV) (Fig. 12).

Fêmea - Medidas: Comprimento cabeça 1,34 (1,2 - 1,47); largura da cabeça $2,57(2,54-2,61)$; comprimento diante dos olhos 0,67 ; distância interocular 1,13 ; distância interocelar $0,5(0,46-0,53)$; diâmetro do ocelo 0,2 . Comprimento dos artículos antenais: I 0,53 , II 1,67 , III 1,47 , IV $2,21(2,14-2,27)$, V 2,01 . Comprimento do pronoto $2,11(2,01-2,07)$; largura do pronoto 5,69 $(5,62$ - 5,76); comprimento escutelo $4,42(4,35-4,48)$; largura escutelo 3,51 $(3,48-3,55)$; comprimento total 10,1 .

Genitália - Margem posterior do esternito VII sinuosa, truncada sobre os gonocoxitos 8 (Gc8). Laterotergitos 8 (La8) destituídos de espiráculos, com margem posterior de contorno triangular, apenas pouco mais longos que os laterotergitos 9 (La9). Gonocoxitos 8 (Gc8) subtrapezoidais; bordos suturais justapostos na metade anterior e amplamente escavados na metade posterior deixando a descoberto o pseudoesternito (gonocoxitos 9). Laterotergitos 9 (La9) claviformes (Fig. 16). Chitinelipsen (ch) presentes. Vias genitais ectodérmicas: 
Ductus receptaculi (DR), na região anterior à área vesicular formando uma espira em torno do espessamento da íntima vaginal (EIV) e com duas vezes e meia o comprimento do ductus na região posterior à área vesicular; área vesicular relativamente pequena com quase o dobro do comprimento dos laterotergitos 9: Capsula seminalis (CS) globóide, com uma única projeção digitiforme, robusta, que pouco ultrapassa a crista anular posterior (cap). (Fig. 17).

Material examinado - SURINAME: 2 machos - Coeroeni, 25/VIII/1959, Geÿskes; 1 fêmea - ibidem, 24/VIII/1959; 1 macho - ibidem, 30/VIII/1959; 1 fêmea - Aucderig (sic), 4/VII/1963. Museu de Leiden.

\section{REFERENCIAS}

GRAZIA, J. 1983. Sobre o gênero Phalaeus Stal, 1862 com a descrição de quatro novas espécies (Heteroptera, Pentatomidae). Revta bras. Ent. 27(2):177-187.

ROLSTON, L.H.; F.J.D. McDONALD; D.B. THOMAS JR. 1980. A conspectus of Pentatomini genera of the Western Hemisphere. Part I (Hemiptera: Pentatomidae). J.N. Y. ent. Soc. 88(2):120-132. 